\title{
Role for the microtubule cytoskeleton in GLUT4 vesicle trafficking and in the regulation of insulin-stimulated glucose uptake
}

\author{
Laura M. FLETCHER, Gavin I. WELSH, Paru B. OATEY ${ }^{1}$ and Jeremy M. TAVARÉ ${ }^{2}$ \\ Department of Biochemistry, School of Medical Sciences, University of Bristol, Bristol BS8 1TD, U.K.
}

Insulin stimulates glucose uptake into adipocytes by promoting the translocation of the glucose transporter isoform 4 (GLUT4) from intracellular vesicles to the plasma membrane. In 3T3-L1 adipocytes GLUT4 resides both in an endosomal pool, together with transferrin receptors, and in a unique pool termed 'GLUT4 storage vesicles' (GSVs), which excludes endosomal proteins. The trafficking of GLUT4 vesicles was studied in living 3T3-L1 adipocytes by time-lapse confocal microscopy of GLUT4 tagged with green fluorescent protein. GLUT4 vesicles exhibited two types of motion: rapid vibrations around a point and short (generally less than $10 \mu \mathrm{m}$ ) linear movements. The linear movements were completely blocked by incubation of the cells in the presence of microtubule-depolymerizing agents. This suggests that a subpopulation of GLUT4 vesicles can exhibit motordriven movements along microtubules. Upon further examination, microtubule depolymerization inhibited insulin-stimulated glucose uptake and GLUT4 translocation to the plasma membrane by approx. $40 \%$, but had no effect on insulin-induced translocation of the transferrin receptor to the plasma membrane from endosomes. We propose that an intact microtubule cytoskeleton may be required for optimal trafficking of GLUT4 present in the GSV pool, but not that resident in the endosomal pool.

Key words: glucose transport, signalling, tubulin, vesicle dynamics.

\section{INTRODUCTION}

A major physiological effect of insulin is the rapid stimulation of glucose uptake into its target tissues, namely muscle and fat. This occurs via the ability of insulin to promote the translocation of intracellular vesicles containing the glucose transporter isoform 4 (GLUT4) to the plasma membrane (reviewed in [1,2]). A detailed molecular description of how insulin promotes this translocation is presently lacking, although some of the components likely to be involved have been identified.

There is a considerable body of evidence favouring the presence of GLUT4 in multiple intracellular vesicle pools. Newly synthesized GLUT4, present in the endoplasmic reticulum and the Golgi complex, undergoes maturation and glycosylation before passing out into at least two types of tubulo-vesicular pool (reviewed in [3]). Morphological analysis by electron microscopy and immunofluorescence, and subcellular fractionation, suggest that a significant proportion of the GLUT4 in adipocytes resides in endosomes that are continuously recycling between the plasma membrane and the cell interior [2-6]. Ablation of recycling endosomes, using diaminobenzidine $/ \mathrm{H}_{2} \mathrm{O}_{2}$ treatment of cells loaded with horseradish peroxidase-conjugated transferrin, leads to significant inhibition of insulin-stimulated GLUT4 translocation $[7,8]$. This suggests that at least some of the insulinresponsive pool of GLUT4 derives from recycling endosomes. Consistent with this, insulin also causes the translocation of other proteins resident in the recycling endosomal system, such as the transferrin receptor and GLUT1 $[2,5,6,9,10]$.

GLUT4 also resides in a compartment that lacks endosomal markers, like the transferrin receptor and GLUT1 $[2,3,5,6]$.
This pool has been called the 'GLUT4 storage vesicle' (GSV) compartment by James and his colleagues, and may contain up to $60 \%$ of the insulin-responsive GLUT4 present in an adipocyte (reviewed in [3,11]). Mathematical modelling data [12] and sucrose-density-gradient centrifugation studies [13] also suggest the presence of at least two distinct insulin-responsive GLUT4containing pools.

The activation of phosphoinositide 3-kinase (PI-3K) by insulin is essential for the translocation of GLUT4 from both the endosomal and GSV pools. We have reported that constitutivelyactive forms of protein kinase $B$ induce the translocation of GLUT4 present in the GSV compartment, but not that of GLUT4 present in recycling endosomes [14]. Thus there appear to be distinct mechanisms operating downstream of PI-3K leading to the translocation of GLUT4 from the GSV and endosomal pools. Furthermore, the vesicle- and targetsynaptosomal protein receptors (SNAREs), vesicle-associated membrane protein 2 (VAMP2) and synaptosomal protein with a molecular mass of $23 \mathrm{kDa}$ (SNAP-23) respectively, are also important for plasma-membrane fusion and targeting of GSVs but not of endosomes [14].

Microtubules play a central role in the cellular transit and trafficking of many different types of vesicle (e.g. secretory vesicles and granules), as well as maintaining the structure and positioning of a number of organelle types (reviewed in [15]). For example, there is evidence for the involvement of microtubules in the organization of both the endoplasmic reticulum and the Golgi complex. Vesicles budding from the endoplasmic reticulum traffic towards the Golgi complex located at the minus end of microtubules in what appears to be a dynein-mediated motor-

Abbreviations used: DMEM, Dulbecco's modified Eagle's medium; GLUT4, glucose transporter isoform 4; IRAP, insulin-responsive aminopeptidase; GSV, GLUT4 storage vesicle; GFP, green fluorescent protein; PI-3K, phosphoinositide 3-kinase; SNAP-23, synaptosomal protein with a molecular mass of $23 \mathrm{kDa}$; SNARE, synaptosomal protein receptor; VAMP2, vesicle-associated membrane protein 2; KRP, Krebs-Ringer phosphate.

1 Present address: PerkinEImer LifeSciences, Perkin Elmer House, 204 Cambridge Science Park, Milton Road, Cambridge CB4 0GZ, U.K.

2 To whom correspondence should be addressed (e-mail j.tavare@bristol.ac.uk). 
driven event [16]. The role of such microtubule structures in GLUT4 trafficking and translocation has not been addressed.

GLUT4 tagged at the N-terminus with green fluorescent protein (GFP) (GFP-GLUT4) resides in both the GSV compartment and in recycling endosomes, and translocates to the plasma membrane in response to insulin with characteristics expected of native GLUT4 $[14,17]$. We previously proposed that GLUT4 vesicles were apparently tethered to an intracellular anchor, and that a major role of the insulin-signalling pathway must be to release GLUT4 vesicles from this tether [17]. In these initial studies, which utilized laser-scanning confocal microscopy, laser toxicity and slow imaging rates (maximum of 1 frame/4 s) hampered our efforts to follow GFP-tagged GLUT4 vesicles in 'real-time'. Confocal imaging using improved Nipkow disk technology to illuminate the cells, rather than single-beam laser scanning, has helped to circumvent this problem. The Nipkow disk contains 20000 pinholes coupled to a second disk with 20000 corresponding microlenses, allowing simultaneous laser excitation of the entire field of view. This now allows subsecond frame-rate imaging at low laser power and with a high signal-tonoise output.

Observations of the characteristics of GFP-GLUT4 vesicle movements in 3T3-L1 adipocytes led us to believe that the cytoskeleton was important in GLUT4 vesicle dynamics. In the present study we demonstrate that a subset of GLUT4 vesicles appear to interact with, and move along, microtubule structures. Furthermore, disruption of the microtubule cytoskeleton, using colchicine or vinblastine, blocks these movements and inhibits insulin-stimulated glucose uptake and GLUT4 translocation. On the other hand, microtubule depolymerization does not affect insulin-stimulated transferrin-receptor translocation. Taken together, our data suggest that microtubules may play an important role in maintaining the organization of the GSV compartment in the basal state and in facilitating its maximal translocation to the plasma membrane in response to insulin.

\section{EXPERIMENTAL}

\section{Materials}

The murine 3T3-L1 fibroblast clone was obtained from the A.T.C.C. (catalogue no. CCL 92.1). Tissue culture reagents were purchased from Gibco BRL (Paisley, Renfrewshire, Scotland, U.K.) or Sigma (Poole, Dorset, U.K.). Unless otherwise stated all other reagents were obtained from Sigma. The plasmid pGFP-GLUT4 has been described elsewhere [17]. 2-Deoxy-[$\left.{ }^{3} \mathrm{H}\right]-$ D-glucose was purchased from Amersham Pharmacia Biotech (Little Chalfont, Bucks., U.K.) and ${ }^{125}$ I-transferrin was purchased from NEN Life Science Products (Hounslow, Middx., U.K.)

\section{Cell culture, adipocyte differentiation and microinjection}

Differentiation of 3T3-L1 fibroblasts into the adipocyte phenotype was performed according to procedures detailed in [17]. The cells were grown in 12-well plates (Corning, Corning, NY, U.S.A.) for transport measurements and surface ${ }^{125} \mathrm{I}$-transferrin binding measurements, or on polylysine-coated $22 \mathrm{~mm}$ glass coverslips for microinjection. Microinjection was carried out using an Eppendorf semi-automatic system; the cells were incubated in Dulbecco's modified Eagle's medium (DMEM) supplemented with $10 \%(\mathrm{v} / \mathrm{v})$ Myoclone-Plus foetal calf serum, $2 \mathrm{mM} \mathrm{NaHCO}_{3}$ and $25 \mathrm{mM}$ Hepes, $\mathrm{pH}$ 7.4. Plasmids were microinjected at $20-100 \mu \mathrm{g} / \mathrm{ml}$. Following microinjection, the cells were incubated at $37^{\circ} \mathrm{C}$ in DMEM containing $10 \%(\mathrm{v} / \mathrm{v})$ myoclone-plus foetal calf serum and were supplemented with 200 units $/ \mathrm{ml}$ benzylpenicillin and $100 \mu \mathrm{g} / \mathrm{ml}$ streptomycin. 3T3-L1 fibroblasts were maintained and microinjected in DMEM containing $10 \%$ (v/v) newborn calf serum, 200 units $/ \mathrm{ml}$ benzylpenicillin and $100 \mu \mathrm{g} / \mathrm{ml}$ streptomycin. In all cases, the cells were maintained for 16-24 h following microinjection to allow expression of protein encoded by the injected plasmid DNA. Before any further manipulation or imaging, the cells were incubated for $3 \mathrm{~h}$ at $37^{\circ} \mathrm{C}$ in serum-free DMEM in the presence or absence of inhibitors, and then in the presence of $200 \mathrm{nM}$ insulin, as detailed in the Figure legends.

\section{Cell incubations and immunofluorescence analysis}

Where the cellular distribution of endogenous GLUT4 was determined, the cells were fixed using $4 \%(\mathrm{w} / \mathrm{v})$ paraformaldehyde for $20 \mathrm{~min}$ at room temperature followed by permeabilization for $45 \mathrm{~min}$ in PBS containing $0.1 \%$ (w/v) saponin and $3 \%(\mathrm{w} / \mathrm{v})$ BSA. All subsequent antibody incubations were performed in PBS containing $0.1 \%(\mathrm{w} / \mathrm{v})$ saponin and $3 \%(\mathrm{w} / \mathrm{v})$ BSA. Cells were stained with monoclonal anti-GLUT4 antibodies (Genzyme, Cambridge, MA, U.S.A.) at $2 \mu \mathrm{g} / \mathrm{ml}$ for $1 \mathrm{~h}$, followed by incubation in a 1:200 dilution of FITC-conjugated goat antimouse IgG (Sigma) for $1 \mathrm{~h}$. For all other immunofluorescence analyses, the cells were fixed in $4 \%(\mathrm{w} / \mathrm{v})$ paraformaldehyde and permeabilized in PBS containing $1 \%(\mathrm{v} / \mathrm{v})$ Triton X-100 for 5 min at room temperature before staining with the appropriate antibodies diluted in PBS containing 3\% (w/v) BSA for $30 \mathrm{~min}$, followed by incubation in a $1: 200$ dilution of the relevant FITCconjugated secondary antibody (Sigma) for $30 \mathrm{~min}$. All antibody incubation steps were followed by extensive washes in PBS.

Microtubule structures were visualized using monoclonal antibodies directed against $\alpha$-tubulin (Serotec, Kidlington, Oxford, U.K.) at $2 \mu \mathrm{g} / \mathrm{ml}$. In other experiments insulin-responsive aminopeptidase (IRAP) was detected using polyclonal anti-IRAP serum (a gift from Dr S. Keller, University of Virginia Health Sciences Center, Charlottesville, VA, U.S.A.) at a dilution of $1: 200$. Alternatively, fixed and permeabilized cells were stained for polymerized actin by incubation in rhodamine-phalloidin (Molecular Probes, Eugene, OR, U.S.A.) at a concentration of $0.1 \mu \mathrm{g} / \mathrm{ml}$ in PBS containing $3 \%(\mathrm{w} / \mathrm{v})$ BSA for $30 \mathrm{~min}$ at room temperature.

\section{Transferrin-Alexa568 uptake assays}

Cells were serum starved for $2 \mathrm{~h}$ in serum-free DMEM and were then incubated for $1 \mathrm{~h}$ in serum-free DMEM, supplemented with $1 \%(\mathrm{w} / \mathrm{v})$ BSA. Subsequently, the medium was replaced with DMEM containing $20 \mu \mathrm{g} / \mathrm{ml}$ fluorescent transferrinAlexa568 (Molecular Probes) for $1 \mathrm{~h}$ at $37^{\circ} \mathrm{C}$. The cells were rapidly and extensively washed in PBS prior to fixing in $4 \%$ (w/v) paraformaldehyde as described above.

\section{Confocal microscopy of fixed cells and image analysis}

Laser-scanning confocal microscopy of fixed cells was performed with a Leica SP inverted confocal imaging spectrophotometer controlled with TCS-NT software (Leica; Lasertechnik, Heidelberg, Germany). Excitation of GFP or FITC-conjugated antibody staining was achieved with a $488 \mathrm{~nm}$ laser, and emission between 500 and $590 \mathrm{~nm}$ was collected. Images of rhodamine or Alexa568 fluorophores were obtained by $568 \mathrm{~nm}$ laser excitation and detection of the emitted light across a 580-665 nm window. Images were collected through the entire cell at $0.5-1.0 \mu \mathrm{m}$ steps 
to produce a $\mathrm{z}$-series that was used to generate a projection image of the cell(s)

Subsecond frame-rate confocal imaging of GFP-GLUT4 in live cells was performed with a Wallac UltraVIEW confocal microscope (PerkinElmer LifeSciences, Great Shelford, Cambridge, U.K.). Images were collected, following $488 \mathrm{~nm}$ laser excitation, by using a FITC emission filter.

Prior to any live confocal microscopy, the cells were incubated in serum-free DMEM for $3 \mathrm{~h}$ with or without drugs, as appropriate. During imaging, the cells were incubated on a heated stage at $37^{\circ} \mathrm{C}$ in a modified Krebs-Ringer phosphate buffer (KRP; $136 \mathrm{mM} \mathrm{NaCl}, 4.7 \mathrm{mM} \mathrm{KCl}, 1.25 \mathrm{mM} \mathrm{MgSO}_{4}$, $1.25 \mathrm{mM} \mathrm{CaCl}_{2}, 5 \mathrm{mM}$ sodium phosphate, $5 \mathrm{mM}$ D-glucose, $2 \mathrm{mM} \mathrm{NaHCO}_{3}$ and $25 \mathrm{mM}$ Hepes, $\mathrm{pH}$ 7.4).

Images were processed with Adobe Photoshop 5.5, Paint Shop Pro (SPSS Inc., Chicago, IL, U.S.A.) and GIF Constructor Set Professional version 2.0 (Alchemy Mindworks). Vesicle movements were traced, and velocities and distances were calculated, using a particle tracking module attached to NIH Image 1.62 (National Institutes of Health, Bethesda, MD, U.S.A.).

In fixed cells, the extent of GFP-GLUT4 translocation to the plasma membrane was quantified by marking a region of interest around the entire exterior and interior faces of the plasma membrane in the confocal plane. The levels of fluorescence intensity within these areas were then computed using TCS-NT software, and the intensity of plasma-membrane localized GFPGLUT4 fluorescence $\left(F_{\mathrm{PM}}\right)$ was expressed as a percentage of total cellular GFP-GLUT4 fluorescence intensity $\left(F_{\mathrm{T}}\right)$. This method corrects for variations in cell shape, size and the expression level of GFP-GLUT4.

\section{Glucose uptake assays}

3T3-L1 adipocytes in 12-well plates were preincubated in serumfree DMEM in the presence or absence of $10 \mu \mathrm{M}$ colchicine or $10 \mu \mathrm{M}$ vinblastine, for $30 \mathrm{~min}$ or $3 \mathrm{~h}$ as appropriate. For the final 15 min the cells were transferred to pre-warmed KRP buffer, also containing the appropriate drugs (total volume per well = $0.45 \mathrm{ml}$ ), after rinsing three times in PBS. Subsequently, where appropriate, cells were treated with insulin for $20 \mathrm{~min}$ at $37^{\circ} \mathrm{C}$. Glucose uptake was quantified by exposing the cells to $50 \mu \mathrm{M} 2-$ deoxy- $\left[{ }^{3} \mathrm{H}\right] \mathrm{D}$-glucose $(1 \mu \mathrm{Ci} / \mathrm{ml})$ for the last $5 \mathrm{~min}$ of the incubation period. Non-specific uptake in basal cells was determined by pretreating the cells for $30 \mathrm{~min}$ with $10 \mu \mathrm{M}$ cytochalasin B to block glucose transporter-mediated uptake prior to assay. At the end of the incubation with 2-deoxy- $\left[{ }^{3} \mathrm{H}\right] \mathrm{D}$-glucose, the cells were washed rapidly three times in ice-cold PBS and were subsequently lysed in $0.5 \mathrm{ml}$ of PBS containing $1 \%(\mathrm{v} / \mathrm{v})$ Triton X-100. The cell-associated radioactivity was then determined by liquid-scintillation counting. All experiments were carried out in triplicate and repeated on at least three separate occasions.

\section{Cell surface ${ }^{125} \mathrm{I}$-transferrin binding assays}

3T3-L1 adipocytes grown and differentiated on 12-well plates were washed three times in PBS at room temperature and were then incubated in $0.5 \mathrm{ml}$ of KRP (pH 7.28) and drugs according to the Figure legends. Insulin $(200 \mathrm{nM})$ was added as appropriate and the cells were then incubated for a further $20 \mathrm{~min}$. The plates were then transferred on to ice and each well was washed twice in ice-cold KRP then incubated in $0.5 \mathrm{ml}$ of ice-cold KRP containing $1 \mathrm{mg} / \mathrm{ml}$ BSA and $20000-40000$ c.p.m. of ${ }^{125} \mathrm{I}-$ transferrin for $2 \mathrm{~h}$ at $4{ }^{\circ} \mathrm{C}$. After this time, the cells were washed three times in ice-cold PBS and were then solubilized in $0.5 \mathrm{ml}$ of $1 \%(\mathrm{v} / \mathrm{v})$ Triton X-100 in PBS. The radioactivity associated with the cell lysate was determined in a $\gamma$-counter. The data were corrected for non-specific binding, which was calculated as the ${ }^{125}$ I-transferrin bound in the presence of an excess of unlabelled human holotransferrin $(1 \mu \mathrm{M})$. All experiments were carried out in triplicate and repeated on at least three separate occasions.

\section{RESULTS}

\section{Time-lapse confocal microscopy of GFP-GLUT4 movements in insulin-stimulated 3T3-L1 adipocytes}

In our previous studies we used laser-scanning confocal microscopy to visualize the dynamics of GFP-GLUT4-containing vesicles in 3T3-L1 adipocytes. While we were able to demonstrate that this chimaera was targeted to bone fide GLUT4 vesicles, we were unable to follow their insulin-dependent translocation to the plasma membrane in real time as a result of laser phototoxicity $[14,17]$. Recent technological developments in Nipkow disk technology, which provides a microlens system for improved optical throughput, allows excitation using lower incident laser levels, thus causing minimal photobleaching and phototoxicity. We have used the Wallac UltraVIEW confocal microscope to image GFP-GLUT4-containing vesicles in 3T3-L1 adipocytes under conditions in which we get virtually no bleaching of the GFP moiety and, as laser toxicity is much less of a problem, greatly improved insulin responses during imaging.

In serum-deprived adipocytes, GFP-GLUT4 is present in vesicles distributed throughout the cytoplasm and concentrated in the perinuclear region. We have previously demonstrated that GFP-GLUT4 shows almost complete co-localization with the resident GLUT4-vesicle protein IRAP and a partial overlap with markers of the endosomal system, such as GLUT1 or transferrin receptors [14].

Adipocytes expressing GFP-GLUT4 were treated on the microscope stage with insulin and imaged at $15 \mathrm{~s}$ intervals over a 30 min period. Figure 1 shows selected stills from a typical experiment (a complete animation of this experiment can be accessed via the URL: http://www.BiochemJ.org/bj/352/ bj3520267add.htm). In the absence of insulin, GFP-GLUT4 was not detectable in the plasma membrane. Upon the addition of insulin, GFP-GLUT4 translocated to the plasma membrane such that a ring of fluorescence was clearly visible within $10 \mathrm{~min}$, and the effect was complete by $20 \mathrm{~min}$.

Close inspection of Figure 1 reveals that the GFP-GLUT4 that translocates to the plasma membrane in response to insulin, derives primarily from the dispersed cytoplasmic pool of peripheral vesicles rather than from the denser perinuclear region, which almost certainly comprises the Golgi complex and associated vesicles.

Inspection of a number $(>10)$ of experiments similar to that illustrated in Figure 1 suggests that there are two subsets of GFP-GLUT4 vesicles: those that are highly dynamic and difficult to image at the lower frame rates and those that remain quite static (see further analysis below). Indeed, many GFP-GLUT4 vesicles move very little during the insulin incubation, but appear to exhibit a gradual decrease in their fluorescence intensity in parallel with an increase in plasma membrane fluorescence (see Figure 1). Other vesicles move quickly from their point of origin and thus disappear between frames. A considerable amount of movement occurs underneath the plasma membrane, with some vesicles transiently attaching to the plasma membrane concomitant with an increase in plasma-membrane fluorescence.

Further understanding of the precise details of the insulindependent translocation will require subsecond frame-rate imaging of vesicle movements during the entire insulin time course. Technically, this proves difficult because, even at the low- 

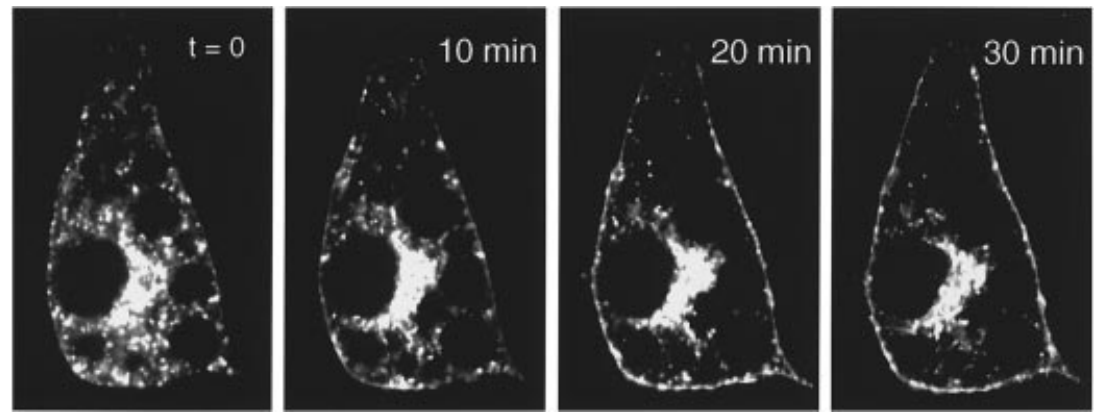

Figure 1 Time-lapse confocal microscopy of insulin-stimulated GFP-GLUT4 translocation in 3T3-L1 adipocytes

A 3T3-L1 adipocyte expressing GFP-GLUT4 was equilibrated in KRP on a heated microscope stage at $37^{\circ} \mathrm{C}$. Insulin was added at $t=0$, and the cell was imaged using a Wallac Ultra VIEW confocal microscope at 1 frame/15 s. During this time, a fine ring of fluorescence becomes visible around the plasma membrane, indicating translocation of GFP-GLUT4 from the intracellular vesicular pool to the plasma membrane. Selected stills are shown at the indicated times, but a full animated sequence can be viewed at http://www.BiochemJ.org/bj/352/bj3520267add.htm.

A
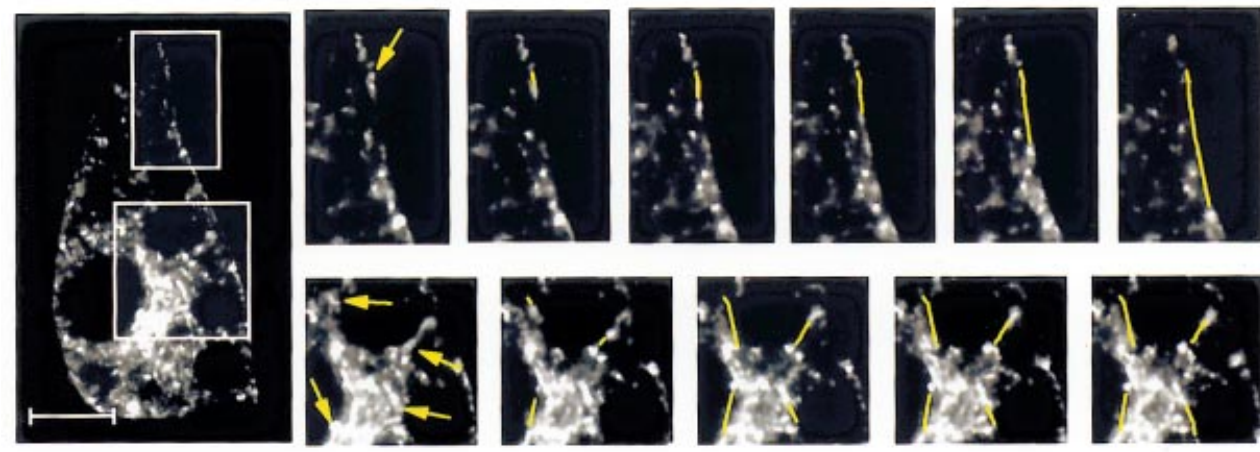

B
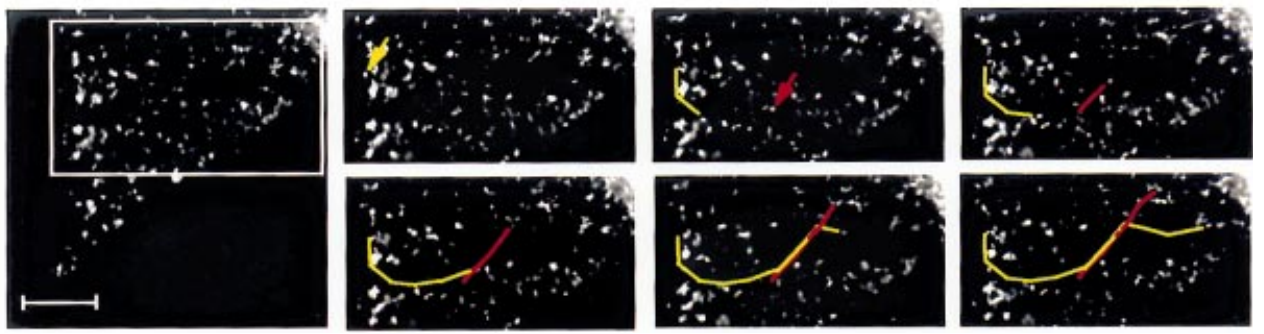

Figure 2 Subsecond frame-rate confocal imaging of GFP-GLUT4 expressed in 3T3-L1 fibroblasts and adipocytes

GFP-GLUT4 was expressed in 3T3-L1 fibroblasts and adipocytes by microinjection. After $24 \mathrm{~h}$ the cells were serum starved for $3 \mathrm{~h}$ prior to imaging using a Wallac Ultra VIEW confocal microscope at approx. 2 frames/s. Selected stills of the boxed regions of interest are shown for an adipocyte (A) and a fibroblast (B). However, the full animated sequences can be found at http://www.BiochemJ.org/bj/352/bj3520267add.htm. The yellow and red lines on the Figures trace the movements of vesicles over a 1 min period, and are discussed in the text. The scale bars represent $10 \mu \mathrm{m}$.

light-level excitation achieved using the Wallac UltraVIEW system, the near continuous illumination of a cell with laser light for up to $20 \mathrm{~min}$ is still toxic to the cells.

Subsecond frame-rate imaging of GFP-GLUT4 vesicle dynamics in 3T3-L1 adipocytes and fibroblasts in the basal state

Given the remarkably dynamic nature of a subpopulation of GFP-GLUT4 vesicles observed in Figure 1, we next used subsecond frame-rate confocal microscopy ( 2 frames/s over a total imaging time of $60 \mathrm{~s}$ ) to examine the movements of individual GFP-GLUT4-containing vesicles in basal 3T3-L1 adipocytes. Selected frames from a region of interest of a representative adipocyte are shown in Figure 2(A) (see a complete animation of this figure at http://www.BiochemJ.org/bj/352/ bj3520267add.htm).

Two types of vesicle movement were clearly evident. The majority of vesicles remained fairly static over this time course, 


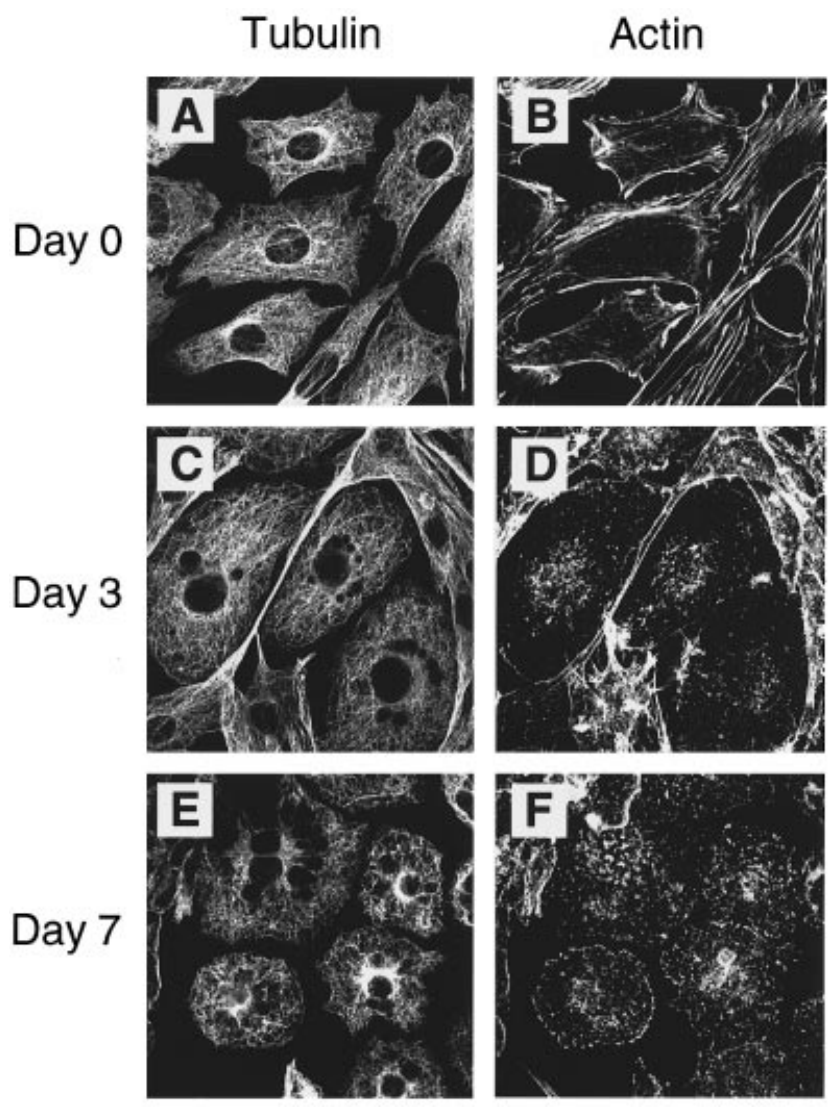

Figure 3 Distribution of microtubules and actin filaments in cells during the differentiation of 3T3-L1 fibroblasts into adipocytes

3T3-L1 fibroblasts were induced to differentiate as described in the Experimental section. The cells were fixed at $0(\mathbf{A}$ and $\mathbf{B}), 3(\mathbf{C}$ and $\mathbf{D})$ or $7(\mathbf{E}$ and $\mathbf{F})$ days and co-stained for the presence of microtubules using anti- $\alpha$-tubulin antibodies $(\mathbf{A}, \mathbf{C}$ and $\mathbf{E})$ or actin filaments using rhodaminephalloidin (B, D and $\mathbf{F})$. Projection images were produced using a Leica laser scanning confocal imaging spectrophotometer as described in the Experimental section.

showing vibrational-type displacements but not moving far from their original location. However, a subset of vesicles are clearly seen to make rapid directed movements over short distances ranging from 5 to $9 \mu \mathrm{m}$ at speeds of approx. $0.8 \mu \mathrm{m} / \mathrm{s}$.

The two populations of vesicles appeared to be interchangeable. For example, a static vesicle could spontaneously begin a rapid linear movement, and subsequently stop and remain static for the remainder of the imaging time. Furthermore, these linear movements were observed both underlying the plasma membrane and deep within the cell. In addition to movements of discrete vesicles, we observed the presence of GFP-GLUT4 in highly dynamic tubular-vesicular structures (this is best observed in the animation).

We also examined the movements of GFP-GLUT4-containing vesicles in 3T3-L1 fibroblasts. While it should be noted that these cells do not express native GLUT4 and are poorly insulin responsive, the data proved highly informative. GFP-GLUT4 was targeted to a highly vesicular intracellular pool in these cells, and was sequestered away from the plasma membrane (Figure $2 \mathrm{~B})$. Images were collected over a period of $1 \mathrm{~min}$ at 1.5 frames $/ \mathrm{s}$. Consistent with our observations in adipocytes, the majority of vesicles remained relatively static for the duration of the experiment, showing only vibrational movements. However, again a small proportion of vesicles made extended linear movements, although these were more frequent and consistently of a significantly greater distance than those observed in adipocytes; ranging between 10 and $29 \mu \mathrm{m}$ at approx. $1.0 \mu \mathrm{m} / \mathrm{s}$. Numerous tubulovesicular structures could be observed in the fibroblasts and these often exhibited movements along linear tracks (Figure 2B).

The vesicle indicated by the yellow line in Figure 2(B) makes a long movement along a curved track, then halts and remains stationary for a period, before making a further extended journey. This is best appreciated by viewing the entire animated sequence at http://www.BiochemJ.org/bj/352/bj3520267add.htm. It is also clear that the tracks along which GFP-GLUT4-containing vesicles travel in 3T3-L1 fibroblasts are stable structures utilized by multiple vesicles. Figure 2(B) shows vesicles indicated by both red and yellow lines that follow the same path for a portion of their extended movement.

As adipocytes are deeper than fibroblasts, it could be argued that vesicles may move out of the plane of view of the confocal section resulting in apparently shorter movements. This is unlikely to be responsible for our inability to observe the longer vesicle movement in adipocytes for two reasons: (1) we have seen none of the longer movements in over 30 separate experiments on adipocytes; and (2) the longer movements are clearly visible by eye in the fibroblasts but not the adipocytes.

\section{Distribution of microtubules and actin filaments during differentiation of 3T3-L1 fibroblasts into adipocytes}

The dynamic linear movements of GFP-GLUT4 vesicles observed in 3T3-L1 adipocytes and fibroblasts are highly characteristic of vesicle movement along cytoskeletal tracks. We next investigated the nature of the tracks that appeared to support the movement of GFP-GLUT4-containing vesicles in 3T3-L1 fibroblasts and adipocytes. Figure 3 shows the distribution of microtubules (visualized with anti- $\alpha$-tubulin antibodies) and actin microfilaments (visualized by staining with rhodaminephalloidin) at selected times during the differentiation of fibroblasts into adipocytes. The images are presented as a projection view of a stack of confocal images taken $0.5 \mu \mathrm{m}$ apart. Viewing images in this way ensures that linear filaments may be reliably observed in deep cells, such as adipocytes, even though they may not lie within a single confocal plane.

In 3T3-L1 fibroblasts, a complex network of microtubules and actin microfilaments can be seen (Figures $3 \mathrm{~A}$ and $3 \mathrm{~B}$ respectively). After initiation of differentiation, the actin microfilaments become extensively depolymerized within $24 \mathrm{~h}$ (results not shown). At day 3 the polymerized actin is seen only as punctate cytoplasmic 'bundles' and as a cortical actin network underlying the plasma membrane, whereas tubulin-containing microtubule structures persist at this time point (Figures $3 \mathrm{C}$ and $3 \mathrm{D}$ ). These redistributions may be necessary to allow the major morphological changes that occur during the differentiation process [18].

Once the cells have attained the adipocyte phenotype (day 7) the cortical actin network is retained and there are few, if any, visible actin filaments in the cytoplasm. However, while the microtubule network has now become considerably more fragmented with the onset of the formation of lipid droplets, shortened microtubules are visible near the periphery of the cell and deep within the cytoplasm (Figures $3 \mathrm{E}$ and $3 \mathrm{~F}$ ). In our previous work, these shortened microtubule structures were not consistently observed [17]. This was probably due to both significant improvements in the resolution of the confocal systems now available to us, and because the images are now viewed as whole-cell projections, as described above. 

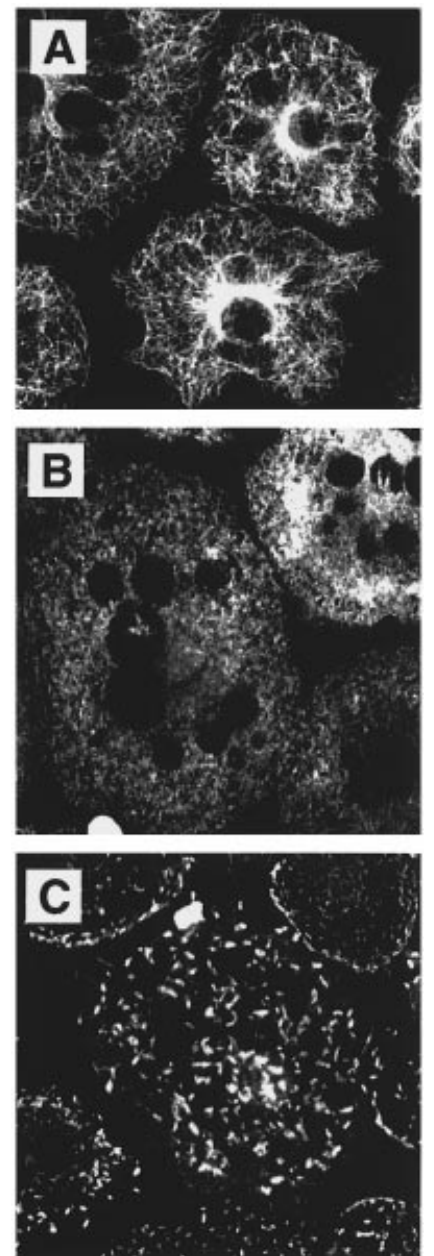

Figure 4 Colchicine and vinblastine cause microtubule depolymerization in 3T3-L1 adipocytes

Differentiated 3T3-L1 adipocytes were incubated for $3 \mathrm{~h}$ in the absence (A) or presence of $10 \mu \mathrm{M}$ colchicine (B) or $10 \mu \mathrm{M}$ vinblastine (C). The cells were fixed and stained for the presence of microtubules using anti- $\alpha$-tubulin antibodies. The images shown are projection images.

\section{Colchicine and vinblastine inhibit GFP-GLUT4 vesicle tracking}

Alterations in the fine structure of tubulin-containing microtubules correlate well with the changes in the dynamics of GLUT4 vesicle tracking that occur upon differentiation of the 3T3-L1 fibroblasts into adipocytes (Figure 2). Furthermore, as cytochalasin D treatment had no effect on the linear movements of GFP-GLUT4 vesicles in 3T3-L1 adipocytes (results not shown), we focused on the role of tubulin in GFP-GLUT4 vesicle movements. To do this we took advantage of colchicine and vinblastine, two structurally unrelated microtubuledestabilizing agents. Colchicine binds free tubulin monomers and acts by disrupting the microtubule lattice at the growing ends, whereas vinblastine inhibits microtubule assembly and promotes tubulin aggregation [19].

Colchicine $(10 \mu \mathrm{M})$ promoted a depolymerization of microtubules in 3T3-L1 adipocytes that was clearly visible within 30 min of addition (results not shown) but required approx. $3 \mathrm{~h}$ to reach completion (Figure 4$)$. Vinblastine $(10 \mu \mathrm{M})$ caused the gradual aggregation of tubulin into short, dense, bundled

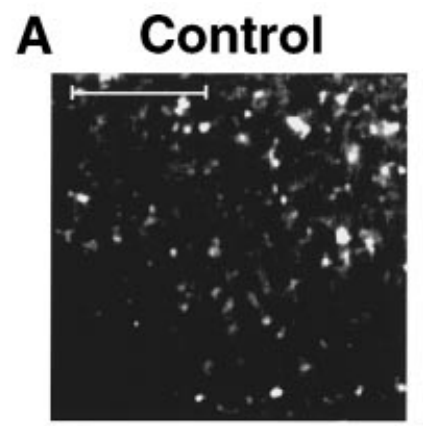

\section{Colchicine}
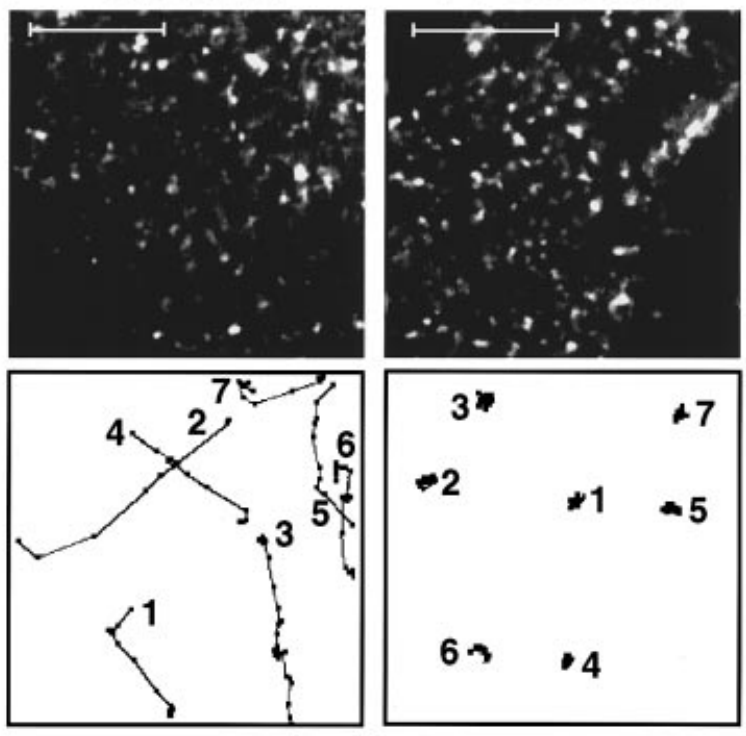

B Control

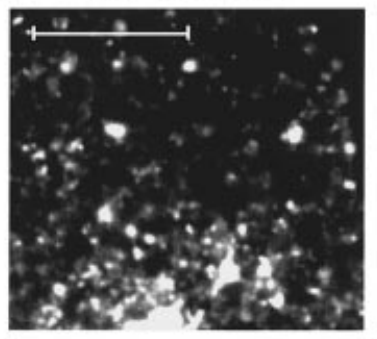

Colchicine
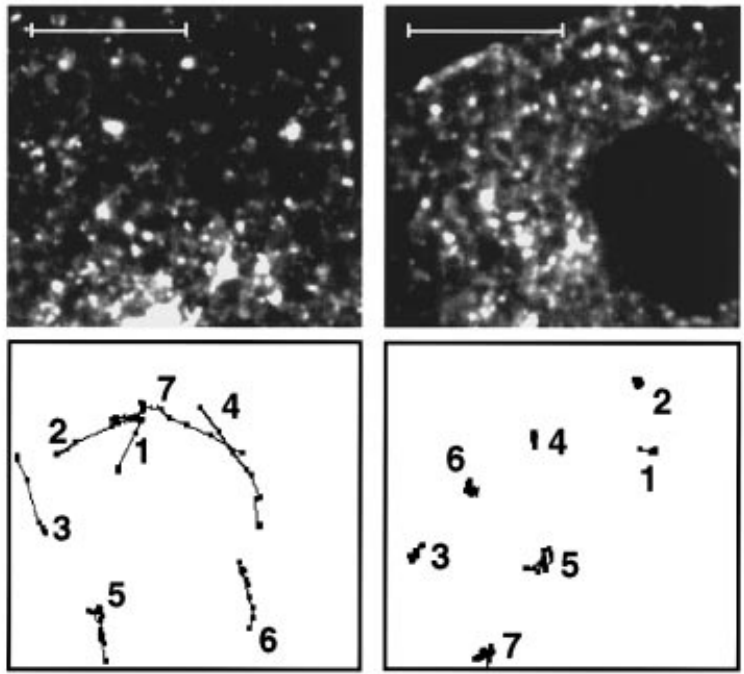

Figure 5 Colchicine treatment blocks GFP-GLUT4 vesicle tracking in basal 3T3-L1 fibroblasts and adipocytes

Differentiated 3T3-L1 fibroblasts $(\mathbf{A})$ or adipocytes $(\mathbf{B})$ were preincubated for $3 \mathrm{~h}$ in the absence or presence of $10 \mu \mathrm{M}$ colchicine as indicated. The cells were imaged using a Wallac Ultra VIEW confocal microscope at 3 frames/s. A selected still of each cell is shown and the movements of the most motile vesicles are traced in the box below each image. The full animated sequences can be found at http://www.BiochemJ.org/bj/352/bj3520267add.htm. The scale bars represent $10 \mu \mathrm{m}$.

structures, and again this effect was significant at $30 \mathrm{~min}$ (results not shown) but was not complete until $3 \mathrm{~h}$ (Figure 4).

Colchicine treatment for $3 \mathrm{~h}$ caused a complete cessation of the linear tracking movements of GFP-GLUT4 vesicles in both the 3T3-L1 fibroblasts (Figure 5A) and adipocytes (Figure 5B). The vibrational motions appeared to be unaffected by colchicine. The characteristics of these movements are best appreciated by viewing the animation at http://www.BiochemJ.org/bj/352/bj3520267add.htm. Vinblastine had an effect that was indistinguishable from that of colchicine (results not shown). 


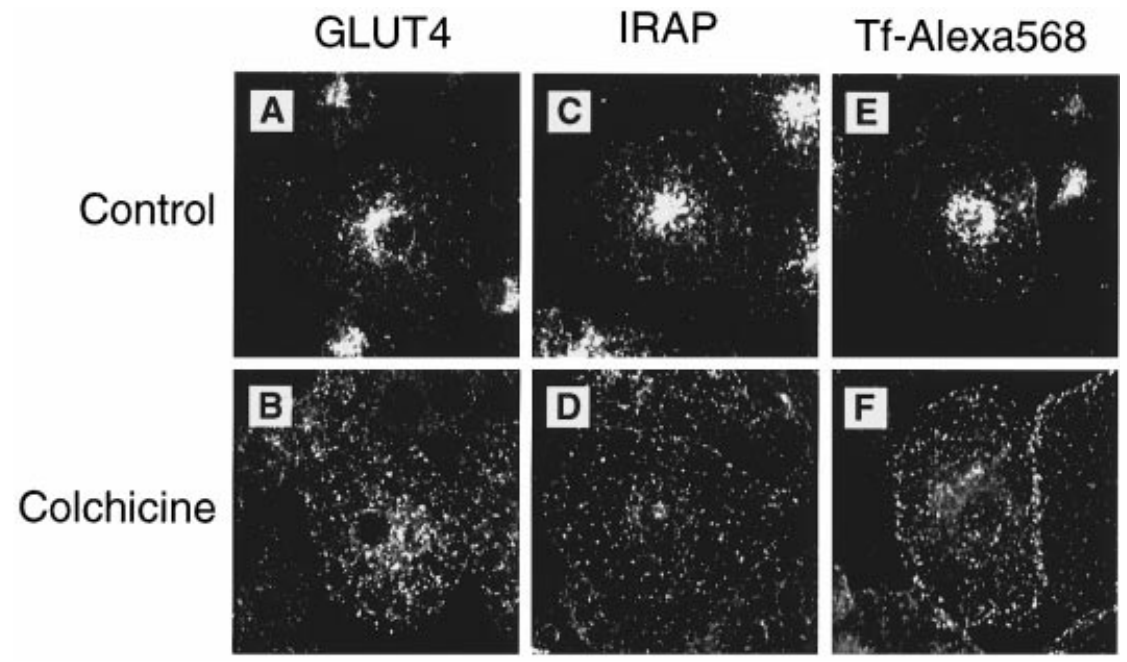

Figure 6 Colchicine treatment causes a redistribution of endogenous GLUT4-, IRAP- and transferrin-containing vesicles in 3T3-L1 adipocytes

Differentiated 3T3-L1 adipocytes were serum starved for $2 \mathrm{~h}$ and then preincubated for $3 \mathrm{~h}$ in the absence $(\mathbf{A}, \mathbf{C}$ and $\mathbf{E})$ or presence $(\mathbf{B}, \mathbf{D}$ and $\mathbf{F})$ of $10 \mu \mathrm{M}$ colchicine. In $(\mathbf{E})$ and $(\mathbf{F})$, transferrinAlexa568 (Tf-Alexa568) was included for the final $1 \mathrm{~h}$ of the preincubation period. The cells were then fixed and stained using anti-GLUT4 antibodies (A and B) or anti-IRAP antibodies (C and D), as described in the Experimental section. Projection images were collected using a Leica laser scanning confocal imaging spectrophotometer. Selected representative cells are shown.

In addition to promoting a cessation of GFP-GLUT4 vesicle tracking, colchicine caused a scattering of native GLUT4 from the perinuclear region to the cell periphery, as observed by staining 3T3-L1 adipocytes with anti-GLUT4 antibodies (Figure $6 \mathrm{~A}$ compared with 6B). The distribution of GFP-GLUT4 (Figure 5) and IRAP (Figure 6C compared with 6D), which colocalize with native GLUT4, were similarly affected. In a similar manner, colchicine prevented the labelling of the perinuclear region of living adipocytes that had been incubated to equilibrium with a fluorescent transferrin analogue (transferrinAlexa568; Figure 6E compared with 6F). In each case, the concentration of fluorescence in the perinuclear region was disrupted. The fluorescence in this perinculear region almost certainly represents GLUT4, GFP-GLUT4, IRAP and transferrin receptors in the Golgi complex and other vesicles that cluster around the microtubule-organizing centre. That this region is disrupted using colchicine is consistent with the known effects of microtubule-interfering drugs on the dispersal of the Golgi complex to peripheral cytoplasmic sites (see [20] for example). Vinblastine had an effect which was indistinguishable from that of colchicine (results not shown). We investigated the possibility that colchicine had an indirect effect on GFP-GLUT4 vesicle movements by dispersing the Golgi complex. To do this, 3T3-L1 adipocytes expressing GFP-GLUT4 were incubated in the presence of brefeldin A $(10 \mu \mathrm{g} / \mathrm{ml})$. This drug collapsed the Golgi complex (as determined by examining the redistribution of GFP-galactosyltransferase, a Golgi-specific marker), but was without effect on the linear movements of GFP-GLUT4 vesicles or their translocation to the plasma membrane in response to insulin, although it did cause significant tubularization of GFPGLUT4 in the perinuclear region (results not shown).

\section{Microtubule depolymerization inhibits insulin-induced glucose uptake and GFP-GLUT4 translocation in 3T3-L1 adipocytes}

Given the apparent role of microtubules in the basal trafficking and cellular localization of GLUT4 vesicles, we examined the effect of microtubule-depolymerizing agents on insulin-induced glucose uptake and GLUT4 translocation in 3T3-L1 adipocytes.
The ability of insulin to induce glucose uptake was assayed by 2deoxy- $\left[{ }^{3} \mathrm{H}\right] \mathrm{D}$-glucose uptake. GFP-GLUT4 translocation was quantified by determining the amount of GFP-GLUT4 found in the plasma membrane as a fraction of the total GFP-GLUT4 expressed in the cell.

Colchicine and vinblastine markedly inhibited insulin stimulation of glucose uptake in a time-dependent fashion, but had no apparent effect on basal levels of glucose uptake. The maximal effect of colchicine and vinblastine occurred after a $3 \mathrm{~h}$ treatment with drugs, with the insulin effect on glucose uptake being reduced by $45 \%$ and $36 \%$, respectively, of that seen in cells not treated with the drugs (Figures $7 \mathrm{~A}$ and $7 \mathrm{~B}$, and results not shown). We also examined the degree of GFP-GLUT4 translocation in response to insulin after a $3 \mathrm{~h}$ treatment with colchicine (Figure 7C). Again, the drug had no effect on basal levels of plasma-membrane GFP-GLUT4, but inhibited the insulin effect by $39 \%$, which is consistent with the extent of the inhibitory effect we observed on insulin-stimulated glucose uptake $(45 \%)$.

Nocodazole, a third structurally-distinct microtubuledepolymerizing drug, has been previously shown to act as a tyrosine kinase inhibitor [21]. While this drug blocked insulinstimulated glucose uptake it also inhibited the activation of protein kinase $\mathrm{B}$, as determined indirectly by measuring the phosphorylation of protein kinase B on serine- 473 by Western blotting with a phospho-specific antibody (results not shown). However, neither colchicine nor vinblastine inhibited either insulin-stimulated serine-473 phosphorylation of protein kinase $\mathrm{B}$ or insulin receptor tyrosine autophosphorylation, excluding the possibility that they block glucose uptake non-specifically by acting on these steps.

\section{Colchicine does not inhibit insulin-stimulated translocation of the transferrin receptor}

GLUT4 exists in two distinct insulin-responsive subcellular compartments (see the Introduction section). One pool is the recycling endosomes where the transferrin receptor is also co-localized, and co-translocates with GLUT4 to the plasma 

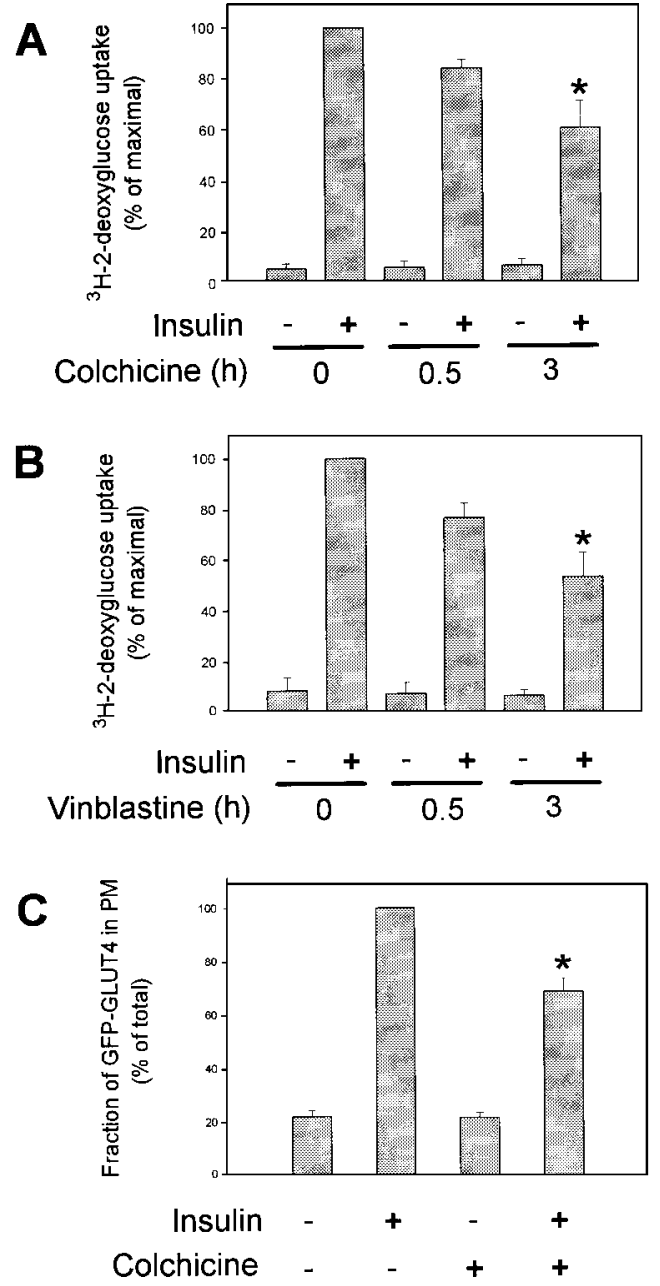

Figure 7 Microtubule depolymerization inhibits insulin-stimulated glucose uptake and GFP-GLUT4 translocation in 3T3-L1 adipocytes

3T3-L1 adipocytes were serum starved for $2 \mathrm{~h}$ and then pretreated with or without $10 \mu \mathrm{M}$ colchicine (A) or vinblastine (B) for $30 \mathrm{~min}$ or $3 \mathrm{~h}$, followed by incubation with or without $200 \mathrm{nM}$ insulin for $20 \mathrm{~min}$, as indicated. 2-Deoxy- $\left[{ }^{3} \mathrm{H}\right] \mathrm{D}$-glucose uptake was determined for the final $5 \mathrm{~min}$ of the insulin incubation. The data shown are taken from three independent experiments (means \pm S.E.M.) and are corrected for non-specific uptake measured in the presence of cytochalasin B. ${ }^{*} P<0.02$ for insulin treatment at $t=0$ when compared with incubation in the presence of colchicine or vinblastine plus insulin at $t=30 \mathrm{~min}$ or $3 \mathrm{~h}$. (C) 3T3-L1 adipocytes were microinjected with a plasmid encoding GFP-GLUT4. After $24 \mathrm{~h}$ the cells were serum starved for $2 \mathrm{~h}$ prior to treatment, as indicated, with or without $10 \mu \mathrm{M}$ colchicine for $3 \mathrm{~h}$ followed by treatment with or without $200 \mathrm{nM}$ insulin for a further $30 \mathrm{~min}$ before fixation with $4 \%(\mathrm{w} / \mathrm{v})$ paraformaldehyde. Images were collected using a Leica laser scanning confocal imaging spectrophotometer and the fraction of total cellular GFP-GLUT4 present in the plasma membrane (PM) was determined as described in the Experimental section. The cells were also stained with anti-( $\alpha$-tubulin) antibodies to confirm the collapse of microtubules (results not shown). The results are pooled from three independent experiments (means \pm S.E.M.) with each experimental condition represented by a minimum of 75 cells. *Indicates a $P$ value of $<0.02$ for the colchicine and insulin treated cells, compared with colchicine-free but insulin treated cells.

membrane in response to insulin. We examined whether colchicine blocked the insulin-dependent translocation of the transferrin receptor to the plasma membrane by measuring the binding of ${ }^{125}$ I-transferrin to cell-surface receptors. As shown in Figure 8 , insulin had an approximately 2 -fold effect on the translocation of the transferrin receptor to the plasma membrane, consistent with previous studies (e.g. see [9]). A $3 \mathrm{~h}$ treatment with colchicine

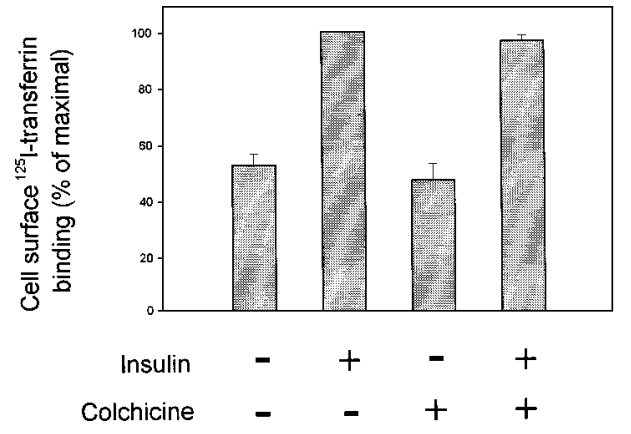

Figure 8 Colchicine treatment has no effect on insulin-induced translocation of the transferrin receptor in 3T3-L1 adipocytes

3T3-L1 adipocytes were serum starved for $2 \mathrm{~h}$ and then pretreated with or without $10 \mu \mathrm{M}$ colchicine for $3 \mathrm{~h}$, followed by incubation with or without $200 \mathrm{nM}$ insulin for $20 \mathrm{~min}$, as indicated. Cell surface ${ }^{125} \mathrm{I}$-transferrin binding was determined as described in the Experimental section. The data shown were taken from three independent experiments (means \pm S.E.M.) and are corrected for non-specific binding, measured in the presence of excess unlabelled human holotransferrin.

was without effect on insulin-stimulated translocation of the transferrin receptor (Figure 8).

Thus while colchicine caused a redistribution of transferrinAlexa568-labelled vesicles found in the perinuclear region, as is the case for GLUT4, GFP-GLUT4 and IRAP (Figures 5 and 6), it does not block the insulin-dependent translocation of the transferrin receptor to the plasma membrane (Figure 8).

\section{DISCUSSION}

Using a GLUT4 construct tagged at the N-terminus with GFP we have been able, for the first time, to monitor the insulinstimulated translocation of GLUT4 to the plasma membrane in 'real-time'. To do this we employed an UltraVIEW confocal microscope which allows low-light-level laser excitation of GFP to help reduce the phototoxicity associated with laser scanning systems. The cell shown in Figure 1 was imaged at subsecond frame rates under basal conditions prior to insulin stimulation (see Figure 2A), demonstrating that toxicity was not a significant problem under these conditions. Figure 1 shows a gradual increase in plasma membrane GFP-GLUT4 content over a 20 min period of insulin stimulation. This and other similar timelapse experiments support the observation that the pool of GLUT4 contributing to the increase in plasma membrane GFP-GLUT4 content are those vesicles residing in the peripheral cytoplasmic compartment of the cell. While GFP-GLUT4 is also heavily expressed in the perinuclear region of the cell (almost certainly this represents compartments associated with the Golgi complex and late endosomes) this contributes only marginally to the pool of GLUT4 that is mobilized by insulin compared with the pronounced depletion of fluorescence from vesicles in the more peripheral cytoplasmic compartment.

Our data also clearly demonstrate that in adipocytes GFPGLUT4-containing vesicles are highly motile and consist of at least two populations with distinct dynamic characteristics. There are those vesicles that are highly motile, and which can only be visualized clearly using subsecond frame-rate imaging (as shown in Figure 2A), and those that remain remarkably static and exhibit only vibrational movements around a single point in space (Figures 1 and 2). Whether insulin induces the more motile population of GFP-GLUT4 vesicles to translocate directly to 
the plasma membrane cannot be determined until the entire insulin time course can be imaged at subsecond frame rates. This still proves to be a challenging proposition due to the requirement for continuous long-term laser illumination of the cells.

Subsecond frame-rate imaging of basal cells reveals that a subset of the more motile GFP-GLUT4 vesicles exhibit dramatic linear tracking motions in 3T3-L1 adipocytes (Figure 2A). These movements are completely blocked by two structurally distinct microtubule-depolymerizing agents, colchicine and vinblastine (Figure 5B, and results not shown), confirming that they are microtubule dependent. Two additional pieces of evidence are consistent with a role for microtubules in GLUT4 vesicle trafficking: (1) we see colchicine-sensitive linear movements of vesicles containing expressed GFP-GLUT4 in 3T3-L1 fibroblasts (Figures 2B and 5A); and (2) the distance over which GFPGLUT4 vesicles move appears to correlate with the integrity of the tubulin cytoskeleton; long microtubules and extended GFPGLUT4 vesicle movements are observed in the fibroblasts, and shorter more fragmented microtubules and consequently shorter GFP-GLUT4 vesicle movements are observed in the adipocytes (compare Figures $2 \mathrm{~B}$ and $3 \mathrm{~A}$, with Figures $2 \mathrm{~A}$ and $3 \mathrm{E}$ respectively). Taken together, the data obtained by expressing GFP-GLUT4 in both fibroblasts and adipocytes strongly support a role for microtubules in GLUT4 vesicle trafficking.

The movements of GFP-GLUT4 vesicles are reminiscent of the dynamics of other types of vesicle, which are also dependent on an intact microtubule cytoskeleton. For example, peroxisomes exhibit long saltatory tracking movements along microtubules, which are blocked by microtubule-depolymerizing agents [22]. Protein export from the endoplasmic reticulum involves the formation of small pre-Golgi vesicle structures which translocate inwards towards the Golgi complex along microtubules by using the minus-end-directed motor complex of dynein/dynactin [16]. This raises the intriguing possibility that motor proteins, such as dynein, are also capable of driving GFP-GLUT4 vesicles along microtubules. As some of the movements we see are in the direction of the Golgi complex (i.e. minus-end directed; e.g. see Figure 2B) this remains a distinct possibility and will require further investigation. Such movements of GFP-GLUT4 vesicles may represent the delivery of GLUT4 back into the Golgi complex for subsequent re-sorting back into the insulinresponsive GLUT4 pool(s).

Several studies have shown that actin microfilament depolymerization caused by cytochalasin $\mathrm{D}$ partially inhibits insulinstimulated glucose uptake in muscle and adipocytes [23-25]. We have confirmed that cytochalasin D partially inhibits insulinstimulated glucose uptake and GLUT4 translocation in adipocytes, but have found that it has no effect on the basal linear movements of GFP-GLUT4 vesicles in either 3T3-L1 fibroblasts or adipocytes (L. M. Fletcher and J. M.Tavaré, unpublished work). This is consistent with the apparent absence of actin stress fibres from the cytoplasm of 3T3-L1 adipocytes (Figure 3F). The apparent disaggregation of actin filaments occurs during a very early stage of 3T3-L1 differentiation, in contrast to changes in microtubule morphology, which occur only after day 3 when fat droplets begin to accumulate. It is important to note that these data do not exclude a role for the actin network in insulininduced translocation of GLUT4, merely that the role of actin is unlikely to be as a cytoskeletal track for the linear GLUT4 vesicle movements we observe.

Interestingly, while the disruption of microtubules inhibits the linear tracking of GFP-GLUT4 vesicles in the basal state, it has no apparent effect on basal glucose uptake (Figures 7A and 7B). In contrast, however, microtubule depolymerization blocks insulin-stimulated glucose uptake and GFP-GLUT4 translo- cation to the plasma membrane by approximately $40 \%$ (Figure 7). These data are consistent with observations in the early $1970 \mathrm{~s}$ which noted that colchicine partially blocked insulin-stimulated glucose oxidation and its conversion into lipids, although its effect on glucose uptake was not determined [26,27].

It is well established that the insulin-responsive GLUT4 exists in at least two distinct insulin-sensitive intracellular pools: the GSVs (which resemble secretory vesicles in neuroendocrine cells); and the recycling endosomes, where it is coexpressed with GLUT1 and the transferrin receptor, which also translocate to the plasma membrane in response to insulin. The fact that microtubule depolymerization does not block insulin-stimulated translocation of transferrin receptors to the plasma membrane strongly suggests that the GFP-GLUT4 residing in recycling endosomes is similarly unaffected by microtubule depolymerization. By inference, this would suggest that the insulindependent mobilization of GLUT4 from the GSV pool is sensitive to disruption by microtubule-depolymerizing drugs. In support of this hypothesis, the partial (approx. $40 \%$ ) inhibitory effect of colchicine and vinblastine on glucose uptake is consistent with published data suggesting that approximately $60 \%$ of GLUT4 resides outside the recycling endosomes that contain GLUT1 and the transferrin receptor $[2,3,8,28]$.

We have previously demonstrated that the insulin-stimulated translocation of GLUT4 from a pool that resembles the GSVs, but not from recycling endosomes, is sensitive to inhibition by Botulinum clostridium toxins that specifically cleave the vesicleand target-SNARE components in fat cells that participate in GLUT4 vesicle targeting to the plasma membrane (VAMP2 and SNAP-23 respectively; [14]). In fact, the partial inhibitory effect of colchicine and vinblastine on insulin-stimulated glucose uptake is also consistent with the partial (approx. 50\%) inhibitory effects of these toxins on insulin-stimulated GLUT4 translocation from the GSV pool $[14,29]$. We have also found that GLUT4 from the GSV pool, but not from the recycling endosomes, could be induced to translocate to the plasma membrane in response to overexpression of a constitutively-active protein kinase $\mathrm{B}$ mutant [14].

Taken together, these data suggest a model in which the insulin sensitivity of the GSV pool of GLUT4 is dependent on the presence of an intact microtubule cytoskeleton, and that GLUT4 from the GSV pool translocates to the plasma membrane in a protein kinase B-, VAMP2- and SNAP-23-dependent manner. Insulin-dependent translocation of GLUT4 from the endosomal pool requires a quite distinct signalling pathway, although, like GLUT4 from the GSV pool, it also requires the activation of PI-3K $[9,14]$. The nature of this signalling pathway requires further investigation. One attractive possibility is that the atypical isoforms of protein kinase $\mathrm{C}(\zeta / \lambda)$ may play a role as these are known to induce the translocation of GLUT1 [30].

Quite how the microtubule cytoskeleton plays a role in modulating insulin-stimulated GLUT4 translocation and the stimulation of glucose uptake is not known. Several putative models can be proposed. For example, GLUT4 vesicles may move towards the plasma membrane in a plus-end directed manner via the use of kinesin motors. Alternatively, microtubules are required for maintaining the GSV compartment in an 'insulinresponsive state'. Depolymerization of microtubules might disrupt the GSV compartment so that either (1) it cannot receive the insulin 'signal', or (2) because disruption to the Golgi region (see Figure 4) may prevent correct sorting of pre-existing and newly synthesized GLUT4 into the GSV pool. These possibilities will require further testing, but it is of interest to note that previous studies on purified GLUT4 vesicles have demonstrated the presence of as yet poorly defined cytoskeletal component(s); such 
components may allow targeting of signalling molecules such as PI-3K to the GLUT4 vesicle [31].

This work was supported by grants from the Medical Research Council and the British Diabetic Association. J. M. T. is a Diabetes U.K. Senior Research Fellow. The confocal microscopy was performed using the MRC Cell Imaging Facility in Bristol that was funded by an Infrastructure Grant from the Medical Research Council. We are grateful to George Banting and Peter Shepherd for useful discussions, and to Susanna Keller for the anti-IRAP antibodies. We thank Michelle Lazenby for her excellent technical assistance.

\section{REFERENCES}

1 Holman, G. D. and Kasuga, M. (1997) From receptor to transporter: insulin signalling to glucose transport. Diabetologia 40, 991-1003

2 Kandror, K. V. and Pilch, P. F. (1996) Compartmentalisation of protein traffic in insulin-sensitive cells. Am. J. Physiol. 34, E1-E14

3 Rea, S. and James, D. E. (1997) Moving GLUT4: the biogenesis and trafficking of GLUT4 storage vesicles. Diabetes 46, 1667-1677

4 Slot, J. W., Geuze, H. J., Gigengack, S., Lienhard, G. E. and James, D. E. (1991) Immuno-localization of the insulin regulatable glucose transporter in brown-adipose tissue of the rat. J. Cell Biol. 113, 123-135

5 Malide, D., Dwyer, N. K., Blanchette-Mackie, E. J. and Cushman, S. W. (1997) Immunocytochemical evidence that GLUT4 resides in a specialized translocation postendosomal VAMP2-positive compartment in rat adipose cells in the absence of insulin. J. Histochem. Cytochem. 45, 1083-1096

6 Malide, D. and Cushman, S. W. (1997) Morphological effects of wortmannin on the endosomal system and GLUT4-containing compartments in rat adipose cells. J. Cell Sci. 110, 2795-2806

7 Livingstone, C., James, D. E., Rice, J. E., Hanpeter, D. and Gould, G. W. (1996) Compartment ablation analysis of the insulin-responsive glucose tranporter (GLUT4) in 3T3-L1 adipocytes. Biochem. J. 315, 487-495

8 Martin, S., Tellam, J., Livingstone, C., Slot, J. W., Gould, G. W. and James, D. E. (1996) The glucose transporter (GLUT-4) and vesicle-associated membrane protein-2 (VAMP-2) are segregated from recycling endosomes in insulin-sensitive cells. J. Cell Biol. 134, 625-635

9 Shepherd, P. R., Soos, M. A. and Siddle, K. (1995) Inhibitors of phosphoinositide 3kinase block exocytosis but not endocytosis of transferrin receptors in 3T3-L1 adipocytes. Biochem. Biophys. Res. Commun. 211, 535-539

10 Tanner, L. I. and Lienhard, G. E. (1987) Insulin elicits a redistribution of transferrin receptors in 3T3-L1 adipocytes through an increase in the rate constant for receptor externalization. J. Biol. Chem. 262, 8975-8980

11 Pessin, J. E., Thurmond, D. C., Elmendorf, J. S., Coker, K. J. and Okada, S. (1999) Molecular basis of insulin-stimulated GLUT4 vesicle trafficking. Location! Location! Location! J. Biol. Chem. 274, 2593-2596

12 Holman, G. D., Lo Leggio, L. and Cushman, S. W. (1994) Insulin-stimulated GLUT4 glucose transporter recycling. A problem in membrane protein subcellular trafficking through multiple pools. J. Biol. Chem. 269, 17516-17524

13 Lee, W., Ryu, J., Souto, R. P., Pilch, P. F. and Jung, C. Y. (1999) Separation and partial characterization of three distinct intracellular GLUT4 compartments in rat adipocytes. Subcellular fractionation without homogenization. J. Biol. Chem. 274, 37755-37762

Received 15 June 2000/25 August 2000; accepted 3 October 2000
14 Foran, P. G. P., Fletcher, L. M., Oatey, P. B., Mohammed, N., Dolly, J. O. and Tavaré, J. M. (1999) Protein kinase B stimulates the translocation of GLUT4 but not GLUT1 or transferrin receptors in 3T3-L1 adipocytes by a pathway involving SNAP-23, synaptobrevin-2, and/or cellubrevin. J. Biol. Chem. 274, 28087-28095

15 Lane, J. and Allan, V. (1998) Microtubule-based membrane movement. Biochim. Biophys. Acta 1376, 27-55

16 Presley, J. F., Cole, N. B., Schroer, T. A., Hirschberg, K., Zaal, K. J. and LippincottSchwartz, J. (1997) ER-to-Golgi transport visualized in living cells. Nature (London) 389, 81-85

17 Oatey, P. B., Van Weering, D. H. J., Dobson, S. P., Gould, G. W. and Tavaré, J. M. (1997) GLUT4 vesicle dynamics in living 3T3 L1 adipocytes visualized with greenfluorescent protein. Biochem. J. 327, 637-642

18 Smas, C. M. and Sul, H. S. (1995) Control of adipocyte differentiation. Biochem. J. 309, 697-710

19 Jordan, M. A. and Wilson, L. (1998) Use of drugs to study role of microtubule assembly dynamics in living cells. Methods Enzymol. 298, 252-276

20 Cole, N. B., Sciaky, N., Marotta, A., Song, J. and Lippincott-Schwartz, J. (1996) Golgi dispersal during microtubule disruption: regeneration of Golgi stacks at peripheral endoplasmic reticulum exit sites. Mol. Biol. Cell 7, 631-650

21 Huby, R. D., Weiss, A. and Ley, S. C. (1998) Nocodazole inhibits signal transduction by the T cell antigen receptor. J. Biol. Chem. 273, 12024-12031

22 Rapp, S., Saffrich, R., Anton, M., Jakle, U., Ansorge, W., Gorgas, K. and Just, W. W. (1996) Microtubule-based peroxisome movement. J. Cell Sci. 109, 837-849

23 Tsakiridis, T., Vranic, M. and Klip, A. (1994) Disassembly of the actin network inhibits insulin-dependent stimulation of glucose transport and prevents recruitment of glucose transporters to the plasma membrane. J. Biol. Chem. 269, 29934-29942

24 Tsakiridis, T., Vranic, M. and Klip, A. (1995) Phosphatidylinositol 3-kinase and the actin network are not required for the stimulation of glucose transport caused by mitochondrial uncoupling: comparison with insulin action. Biochem. J. 309, 1-5

25 Wang, Q. H., Bilan, P. J., Tsakiridis, T., Hinek, A. and Klip, A. (1998) Actin filaments participate in the relocalization of phosphatidylinositol 3-kinase to glucose transportercontaining compartments and in the stimulation of glucose uptake in 3T3-L1 adipocytes. Biochem. J. 331, 917-928

26 Loten, E. G. and Jeanrenaud, B. (1974) Effects of cytochalasin B, colchicine and vincristine on the metabolism of isolated fat cells. Biochem. J. 140, 185-192

27 Soifer, D. (1970) Insulin and microtubules in rat adipocytes. Science 172, 269-271

28 Robinson, L. J. and James, D. E. (1992) Insulin-regulated sorting of glucose transporters in 3T3-L1 adipocytes. Am. J. Physiol. 263, E383-E393

29 Chen, F., Foran, P., Shone, C. C., Foster, K. A. and Dolly, J. O. (1997) Botulinum neurotoxin $B$ inhibits insulin-stimulated glucose uptake into 3T3-L1 adipocytes and cleaves cellubrevin unlike Type A Toxin which failed to proteolyze the SNAP-23 present. Biochemistry 36, 5719-5728

30 Bandyopadhyay, G., Standaert, M. L., Zhao, L., Yu, B., Avignon, A., Galloway, L., Karnam, P., Moscat, J. and Farese, R. V. (1997) Activation of protein kinase C ( $\alpha, \beta$ and $\zeta$ ) by insulin in $3 T 3 / L 1$ cells. Transfection studies suggest a role for PKC- $\zeta$ in glucose transport. J. Biol. Chem. 272, 2551-2558

31 Clark, S. F., Martin, S., Carozzi, A. J., Hill, M. M. and James, D. E. (1998) Intracellular localization of phosphatidylinositide 3-kinase and insulin receptor substrate-1 in adipocytes: Potential involvement of a membrane skeleton. J. Cell Biol. 140, 1211-1225 